El método STEM aplicado a la enseñanza del Derecho. Experiencia en el curso de Procesos Universales I.
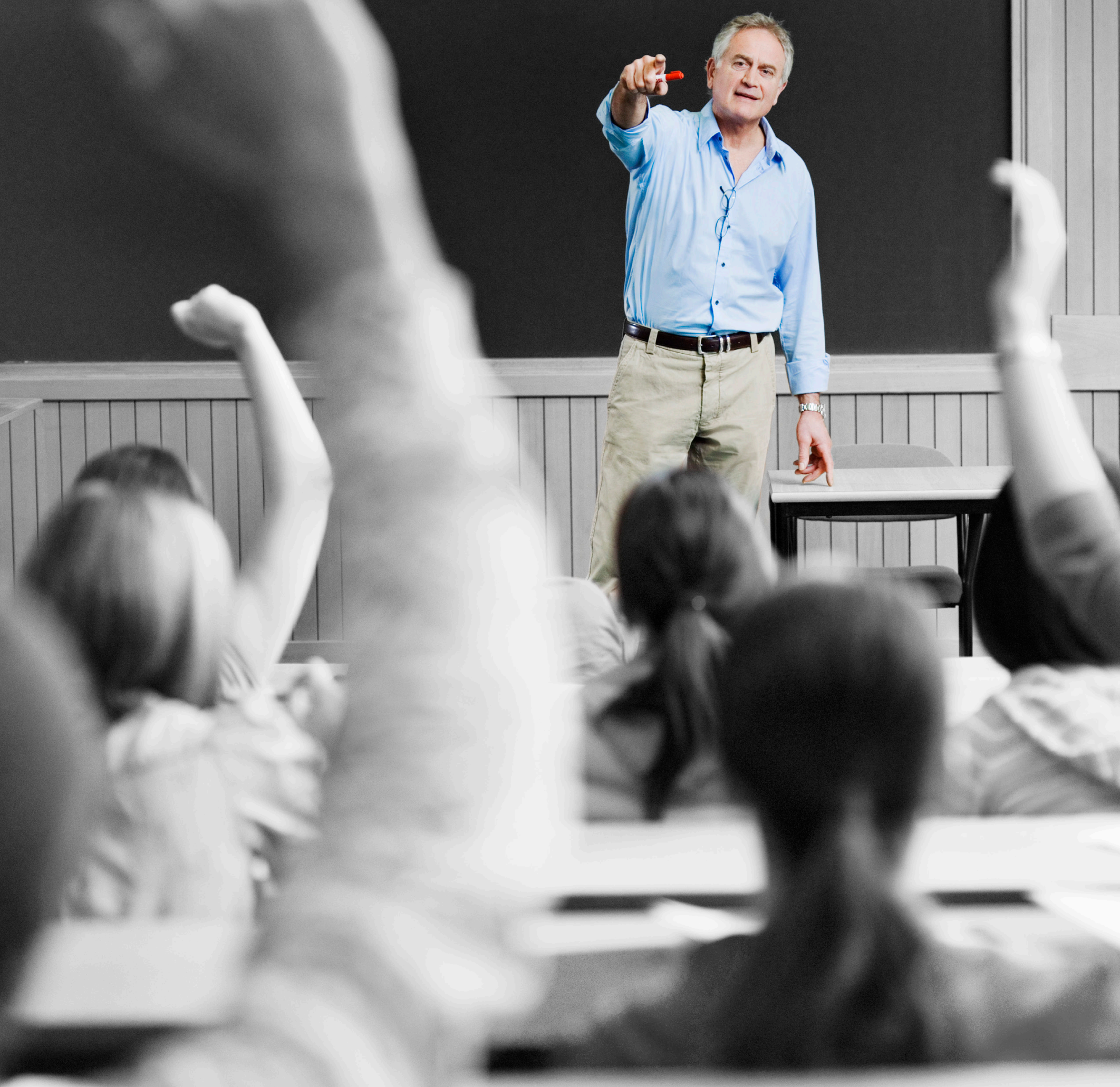


\title{
EI método STEM aplicado a la enseñanza del Derecho. Experiencia en el curso de Procesos Universales I.
}

\author{
M.Sc. Cecilia Ivette Villalobos Soto. \\ Carrera de Derecho. \\ Universidad Fidélitas
}

\begin{abstract}
For many years, Law education has been focused on behavioral teaching-learning models, in which the figure of the teacher plays a central role, he teaches the class, selects the materials to study, clarifies doubts and decides the evaluation way, frequently based on memory. For their part, students have a secondary role, they receive the knowledge transmitted by the teacher and then they use it on the exam. This type of learning does not impact the student and it is quickly forgotten. A radical change is required in our teaching-learning model.
\end{abstract}

The STEM approach is a fundamental tool for this change, since it prepares the student to solve real problems, innovate, learn to learn and continue applying knowledge in his professional life. This approach is ideal for teaching Law. This proposal describes, step by step, the procedure followed to implement the STEM approach in the Universal Processes I course at Fidélitas University Law Career.

We can conclude that is totally feasible to apply the STEM approach in Law education. It is important to keep in mind that for the success of the process, the following factors must be strengthened: teamwork, research, adaptation to changes and use of technology.

Keywords: STEM, Law Education, Active Learning

\section{Introducción}

El Derecho es una ciencia que evoluciona constantemente y como tal su enseñanza debe adaptarse al cambio de los tiempos.

Durante muchos años la enseñanza del Derecho ha estado centrada en modelos de enseñanza-aprendizaje conductivistas, en donde el docente es el personaje central y el estudiante un personaje secundario. La clase gira alrededor del docente, quien imparte la clase, indica los materiales a estudiar y aclara las dudas. Por su parte los estudiantes, se limitan a tomar apuntes, memorizan los contenidos y luego los repiten en sus exámenes. El estudiante obtiene una buena nota si logra repetir en la mejor forma posible los conocimientos impartidos por el docente.

La evaluación de los contenidos dentro de estos modelos de enseñanza, se basa generalmente en métodos memorísticos. Es común que el docente utilice exámenes de marcar con equis, exámenes de respuesta corta, exámenes de desarrollo y exámenes orales entre otros. En todas estas técnicas de evaluación el estudiante se limita a utilizar su memoria y repetir contenidos. El problema es que en poco tiempo olvidan los conocimientos. Incluso cuando el docente utiliza como evaluación la aplicación de casos prácticos, estos se basan en hechos simulados y no en la realidad que rodea a los alumnos.

Un aprendizaje basado únicamente en la memoria no produce un cambio real en el estudiante ni lo prepara para su futura vida profesional.

En muchos casos el docente se siente satisfecho cuando el alumno repite el conocimiento tal y como él lo impartió, pero realmente no se interesa en verificar si el aprendizaje se completó y produjo un cambio significativo en el estudiante. En este sentido, se debe tener presente, que el aprendizaje debe ser significativo, según nos indica Cruz [1] el aprendizaje significativo "... es una forma de aprender con sentido, utilizando la percepción, con referentes en la realidad, con el uso extensivo de la comprensión, con aplicabilidad inmediata; es aquel que llama la atención y despierta el interés del que aprende, que se incorpora a la persona y por lo tanto no se olvida"

En el aprendizaje memorístico y repetitivo, el alumno se limita a "repetir" los contenidos recibidos durante el curso, sin mayores aportes de su parte. Es importante señalar que dentro de la taxonomía de Bloom el estudiante estaría utilizando el nivel de pensamiento de orden inferior: "recordar". 
Hoy en día el mercado laboral requiere de profesionales diferentes, abogados que dominen el conocimiento, pero que a su vez sepan aplicarlo a situaciones cambiantes. Se requiere de profesionales que se adapten al constante cambio y que también dominen la tecnología. En este sentido es importante tener presente que [2] “...la tecnología hace nuestra forma de vivir cada vez más sencilla pero a la vez estos avances están originando impactos sociales y culturales inimaginables. La automatización y la robótica están generando un nuevo fenómeno conocido como "desempleo tecnológico". Las tecnologías de hoy van a hacer desaparecer oficios que nunca hubiéramos pensado que podrían ser reemplazados por máquinas.”

En el campo del Derecho ya se han dado intentos en "robotizar" la aplicación del Derecho a casos concretos. Así en Estonia, se trabaja en un proyecto de "jueces robot", el cual se encuentra en una fase de experimentación, [3] “... Su aplicación funcionará de la siguiente manera: las dos partes cargarán sus documentos e información relevante del caso en una plataforma, donde la IA tomará una decisión que pueda ser apelada por un juez "humano".

Este no es el único caso ya que en algunos estados de los Estados Unidos, se utilizan algoritmos que ayudan a recomendar sentencias penales a los jueces. Actualmente "El caso más conocido es el del chatbox impulsado por DoNotPay, con sede en Reino Unido, que ayudó a anular 160,000 multas de aparcamiento en Londres y Nueva York en los últimos años.” [3]

Sin embargo, en nuestro país está muy lejana esta posibilidad y los abogados son necesarios en muchos ámbitos. Sin embargo, es innegable que se requiere de un nuevo profesional en Derecho. Este abogado del siglo XXI, debe saber trabajar en equipo, conocer de tecnología y estar preparado para los cambios constantes en la aplicación del conocimiento. Por lo tanto, es urgente cambiar el modelo de enseñanza-aprendizaje del Derecho a fin de formar a los nuevos profesionales que requiere la sociedad.

\section{Propuesta de Enfoque STEM para la enseñanza del Derecho.}

Tomando en consideración la necesidad de un cambio toral en la enseñanza del Derecho, es que se propone el enfoque Stem como una nueva herramienta en la enseñanzaaprendizaje del Derecho.

Es importante indicar que nuestra propuesta toma en consideración varios puntos esenciales. En primera instancia, los estudiantes que actualmente llenan las aulas han cambiado. La tecnología hace de ellos personas pensantes, con acceso ilimitado a la información y abiertas además a nuevas estrategias educativas. Por otra parte, el rol pasivo ya no combina con ellos y se requiere que tomen un rol protagónico en su proceso de formación académica.
Por otra parte, es importante tener claro cuál debe ser la función del docente universitario dentro del nuevo modelo. Actualmente su función tradicional está relacionada como el modelo conductual. Según expone Cruz [4] "Se piensa con frecuencia que la esencia del trabajo del docente es enseñar, pero dicha concepción es propia de los modelos centrados en el profesor y en su desempeño. Podemos analizar esta concepción de modelo educativo en las actividades realizadas por el profesor y por el alumno en el Modelo Centrado en la Enseñanza, el profesor explica los temas en clase, expone sus conocimientos, encarga tareas, elabora exámenes y califica; mientras que el alumno atiende a las explicaciones, trata de adquirir los conocimientos, realiza tareas, se prepara para sus exámenes y aprueba o reprueba."

En el modelo educativo que se propone, la función del docente difiere, para Díaz-Barriga y Hernández [5], "El trabajo del docente no es enseñar, el trabajo del docente es propiciar que sus alumnos aprendan" Esta concepción se adapta plenamente a nuestra propuesta.

El uso de la tecnología como herramienta de enseñanzaaprendizaje es esencial. Los jóvenes de hoy ven la tecnología como algo normal y hasta esencial en sus vidas, ya que conviven con ella gran parte del día. Desde este punto de vista, el docente debe aprender a utilizar la tecnología en actividades educativas y no verlo como un simple distractor. La presencia en el aula de teléfonos inteligentes o Tablet facilitarán el cambio.

El trabajo en grupos debe sustituir al trabajo individual, especialmente utilizando la modalidad de grupos colaborativos. Para Glinz [6] " La educación en la actualidad requiere del trabajo de grupo. En las actividades de enseñanza-aprendizaje, el trabajo colaborativo o cooperativo (términos utilizados indistintamente) conforma uno de los principales elementos. Los proyectos innovadores que usan técnicas de enseñanza aprendizaje involucran esta modalidad de trabajo en la que el ser que aprende se forma como persona." Dicha modalidad de trabajo grupal beneficia a todos, ya que obliga a los integrantes a trabajar activamente y a dar un rendimiento comprobable. En el mundo moderno, los profesionales en Derecho deben trabajar en equipo ante la complejidad de los casos que enfrentan día con día. De esta manera aprender a trabajar en grupos desde la Universidad es fundamental.

En resumen, nuestra propuesta es realizar un cambio completo en el modelo educativo de la enseñanza del Derecho. Se requiere un enfoque que tome en consideración el cambio tecnológico, el nuevo rol del estudiante dentro y fuera del aula y asimismo la nueva función del docente. Dicho enfoque debe también integrar el trabajo grupal colaborativo. Desde esta perspectiva el enfoque STEM en la enseñanza del Derecho resulta una herramienta interesante. 


\section{El enfoque STEM aplicado a la enseñanza del Derecho.}

El sistema de educación STEM o como también se le denomina enfoque STEM, está íntimamente relacionado con el significado del acrónimo que integra su nombre. En inglés sería "Science, Technology, Engineering and Mathematics". El enfoque STEM consiste en “...un enfoque científico integrado según el cual los cuatro campos de la Ciencia, Tecnología, Ingeniería y Matemáticas forman un todo donde los elementos interactúan y se afectan unos a otros". [7]

Inicialmente este enfoque de educación, se aplicó a las materias relacionadas con el acrónimo que integra su nombre. Sin embargo, hoy existe una fuerte tendencia a aplicarlo a otras ramas del conocimiento humano, y en nuestro caso al Derecho.

Según indica Rodríguez [8] en el nivel uno, "Es una filosofía educativa: educar para la vida real, para la innovación, para seguir aprendiendo."

En cuanto a los beneficios que trae esta filosofía educativa, nos indica Rodríguez [8] "Prepara al estudiante para enfrentar el cambio: para solucionar problemas reales, para innovar y para que aprenda a aprender." Estos son precisamente los beneficios que se busca obtener al implementar el enfoque Stem en los cursos de Derecho.

Para introducir el cambio en las diferentes materias de la carrera de Derecho, se debe hacer énfasis en el trabajo en equipo, el uso de la tecnología en el proceso de enseñanza e implementar el cambio del rol que cumplen en el proceso educativo tanto el docente como los alumnos.

En la Carrera de Derecho de la Universidad Fidélitas ya se han ido dando pasos en la implementación del Enfoque STEM a través del uso obligatorio del sitio virtual de la universidad, el uso de las bases de datos virtuales y a través de la inclusión de los estudiantes de Derecho en el curso de Administración de proyectos. Es importante indicar que este último curso se imparte para todas las carreras que se imparten en la Universidad y en el trabajo bajo el enfoque multidisciplinario.

\section{Implementación del enfoque STEM en los cursos de la carrera de Derecho.}

Siguiendo los puntos más relevantes desarrollados en la presente ponencia, se pretende demostrar que el enfoque STEM es aplicable a la enseñanza- aprendizaje y evaluación de los cursos de la carrera de Derecho. En nuestro caso se toma como base el curso de Procesos Universales I, sin embargo, es importante dejar claro que siguiendo los pasos indicados es posible su implementación en otros cursos. En este sentido es importante mencionar que mientras se escribía la presente ponencia, se implementó la técnica en el curso de Derecho de Familia I, pero eso implicará un nuevo documento con los resultados y recomendaciones.

Dicho curso trata del tema de las sucesiones o procedimientos que se tramitan a la muerte de una persona con el fin de repartir la totalidad del patrimonio del muerto entre sus acreedores, herederos y legatarios. Se ha escogido en particular este curso ya que involucra un hecho de la vida real común a todas las personas como es la muerte de un familiar. Este último punto facilitará la implementación de la técnica Stem dentro del curso y la búsqueda del problema a resolver.

a. Metodología a utilizar.

La metodología de enseñanza del STEM, según nos indica Rodríguez [8] se basa en proyectos realizados en grupos interdisciplinarios, a los cuales se les da un problema a resolver. El problema será seleccionado por el docente y debe ser actual y con relevancia práctica.

A través de la implementación de esta metodología, los estudiantes no solo aprenderán los contenidos de la disciplina del curso, sino que también aprenderán habilidades blandas, tales como: "pensamiento inductivo, pensamiento deductivo, pensamiento crítico, toma de decisiones, problema- solving skills, trabajo en equipo, manejo del tiempo, manejo de la tarea, liderazgo, pensamiento creativo, aprender a aprender...". [8].

En igual sentido Castro [9] señala que las habilidades blandas más importantes para los profesionales en Costa Rica, son actualmente la “... buena comunicación y organización, trabajo en equipo, pensamiento crítico, sociabilidad, creatividad y habilidades interpersonales de comunicación en general. Todas ellas son aplicables a los profesionales en Derecho.

Otro elemento fundamental del enfoque STEM es la utilización de la investigación dentro del proceso educativo. Con base en la misma se establece el PBL (Problem- Based Learning).

Ahora bien, tanto la metodología STEM como la investigación resultan fundamentales en la enseñanza del Derecho. Los problemas que a diario enfrentan los abogados en la época actual requieren de un profesional formado bajo esta perspectiva. A través del enfoque STEM los estudiantes de Derecho podrán aprender a indagar, a preguntar y a validar el conocimiento adquirido. Lo anterior promoverá el pensamiento crítico, el pensamiento inductivo, el pensamiento creativo, lo cual favorece la formación en habilidades blandas [8]

b. La investigación como metodología de enseñanza dentro del Enfoque STEM. 
Dentro del enfoque STEM la investigación resulta una adecuada metodología de enseñanza, ya que obliga al estudiante a indagar, a descubrir el conocimiento y a aplicarlo correctamente.

Para implementar la investigación dentro de los diferentes cursos que se imparten en la carrera de Derecho, es importante tener presente, los siguientes puntos, tal y como nos expone Rodríguez [8]:

- Se debe identificar el problema a resolver.

- Se deben formular los diferentes escenarios ( hipótesis)

- Los estudiantes deben saber preguntar y el profesor debe saber responder.

- Se debe obtener la información y la prueba de los escenarios.

- Seguidamente se procede al análisis de la información.

- Se sacan conclusiones y

- Finalmente se redacta y se presenta un informe.

c. Evaluación bajo el enfoque STEM.

La evaluación es fundamental en toda actividad académica. Al respecto Díaz-Barriga y Hernández [5] que "...la evaluación es parte integral de una buena enseñanza, de hecho podríamos decir sin ningún menoscabo que no es posible concebir adecuadamente a la enseñanza sin la evaluación."

En el enfoque STEM, el objetivo de la evaluación es: “....determinar el grado de comprensión y la capacidad que el estudiante tiene para aplicar el conocimiento al problema real planteado, así como el aprendizaje y puesta en práctica de habilidades blandas". [8].

En cuanto a las técnicas de evaluación que se pueden utilizar en el modelo PBL, según Rodríguez [8] están: Exámenes escritos-preguntas de escogencia única, exámenes escritos- preguntas de PBL- escenarios, escala de evaluación por compañeros- habilidades blandas, escala de autoevaluación- habilidades blandas, escala de evaluación por el profesor- habilidades blandas, bitácora, presentación informe de solución del problema- grupal.

En el enfoque STEM el docente sabe cuándo se logran los objetivos propuestos, según indica la profesora Neus Sanmarti, profesora emérita de Universidad Autónoma de Barcelona, cuando “... comprobamos que saben activar las ideas que aprendieron, que saben cómo afrontar la resolución de los problemas que se van encontrando, que saben trabajar en equipo, que saben reconocer cuando no lo hacen bien y toman buenas decisiones de mejora". [10]

d. Planeación de la actividad a evaluar.

En esta fase se pasa de la teoría a la práctica. El docente implementa el tema que se desarrollara bajo el enfoque STEM y planifica su aplicación y evaluación.

Para Rodríguez [8], se deben seguir los siguientes pasos:

- Se toma como base el programa del curso.

- Se comienza llevando a cabo simulacros en clase, lo cual le permite al docente conocer cómo trabajan los estudiantes en grupo, lo cual facilitará la conformación de los futuros grupos de trabajo.

- Se forman los grupos para la experiencia PBL.

- Se informa a los estudiantes sobre el valor de la bitácora ( portafolio)

- Se asigna el caso/ problema.

- Se realizan sesiones de tutoría.

- Se evalúan los contenidos y las habilidades blandas adquiridas.

e. Aplicación de la metodología STEM.

Una vez planificada la actividad se procede a la implementación de la actividad y a la organización de los estudiantes. Rodríguez [20] indica que deben tenerse presente los siguientes puntos para correcta aplicación de la técnica de STEM:

- Tutoría.

- Evaluación de dominio de contenidos y de habilidades blandas: del producto y del proceso.

- Evaluación de cómo formulan preguntas.

- Docente debe contestar con "Sín" o "No".

- Evaluación de hipótesis o escenarios y del proceso de construcción de esos escenarios.

- Evaluación del uso de recursos e información para validación de los escenarios.

- Revisión y evaluación de bitácora: proceso. 


\section{Conclusiones:}

Por muchos años la enseñanza del Derecho ha estado basada en modelos conductivistas en donde el docente es el personaje central y los estudiantes ocupan rol secundario.

En la evaluación de los contenidos generalmente se utilizan métodos basados en la memoria.

Los estudiantes que actualmente ocupan nuestras aulas han cambiado y requieren de un nuevo modelo de enseñanzaaprendizaje. En este momento histórico la sociedad requiere de abogados que no solo manejen los contenidos teóricos sino que cuenten además con habilidades blandas tales como trabajo en grupo, investigación, adaptación a los cambios, dominio de la tecnología y otros.

El enfoque STEM en la enseñanza del Derecho, nos permite preparar desde las aulas universitarias futuros abogados que se adapten mejor al cambio de los tiempos y que logren incorporarse a un mercado de trabajo exigente. El objetivo que se persigue al utilizar el enfoque STEM en la formación de futuros abogados es prepararlos para solucionar problemas reales, innovar y aprender a aprender.

Los roles del docente y del alumno deben cambiar. El rol del docente ya no será simplemente enseñar, sino propiciar que los alumnos aprendan a través de actividades motivantes que los obliguen a investigar. Por su parte los estudiantes toman un rol activo. A través de la investigación, la tecnología y el trabajo grupal aprenden a aprender y a forzarse como futuros profesionales.

La presente ponencia desarrolla la necesidad de establecer el enfoque STEM en todos los cursos de la carrera de Derecho de la Universidad Fidélitas.

El cambio no será fácil y requiere de mucha capacitación a los docentes y de motivación tanto a los estudiantes como a los docentes. El camino apenas se inicia, lo importante es seguir con su implementación paulatina y evidenciar los resultados más adelante a través de nuevas ponencias.

La presente ponencia hace referencia a la implementación del enfoque Stem en el curso de Procesos Universales I, pero es importante mencionar que ya se ha iniciado su implementación en el curso de Derecho de Familia I y los estudiantes muestran su satisfacción por el cambio.

\section{Referencias.}

[1] Cruz, R. (2008) El aprendizaje significativo ¿qué es y cómo se logra? Recuperado de https://www.elsiglodetorreon. com.mx/ notici a/383494.el-aprendizaje-significativo-quees-ycomo- se-logra.html

[2] STEM Education Colombia. (2019) Qué es STEM? Recuperado de: https://www.stemeducol.com/que-es-stem
[3] The Technolawgist. (2019) Estonia se prepara para tener "jueces robot" basados en inteligencia artificial. Obtenido de: https://www.thetechnolawgist.com/2019/06 /12/ estonia-se-prepara-para-tener-jueces-robot-basadoseninteligencia- artificial/

[4] Cruz, R. (2008) El aprendizaje significativo ¿qué es y cómo se logra? Recuperado de https://www.elsiglodetorreon. com.mx/noticia/383494.el-aprendizaje-significativoquees-y-como-se-logra.html

[5] Díaz- Barriga, F. y Hernández, G. (2002) Estrategias docentes para un aprendizaje significativo. Una interpretación constructivista. México, México: Mc Graw Hill.

[6] Glinz, P. (2005) Un acercamiento al trabajo colaborativo. Revista Iberoamericana de Educación. Vol. 36 Núm. 7 Número especial. México. Recuperado d e : https://rieoei. org/RIE/article/view/2927

[7] STEM las nuevas Tecnologías al Servicio de la Educación actual. (s.f.e) Qué es el sistema STEM en educación. Recuperado de: https://www.thetechnolawgist. com/2019/06/12/estonia-se-prepara-para-tenerjuecesrobot-basados-en-inteligencia-artificial/

[8] Rodríguez, R. (2019) Enfoque STEM. Un cambio. Universidad Fidélitas. (Documento en Power point) San José, Costa Rica.

[9] Castro, R. (2018). Habilidades blandas: aliadas necesarias para el profesional. Recuperado de: https://www. primeraplana.or.cr/es/Contorn o/Habilidades_blandas aliadas_necesarias_para_el_profesional/

[10] Educación 3.0 (2019) 10 claves para implantar la educación en STEAM en el aula. Recuperado de: https:// www.educaciontrespuntocero.com $/ \mathrm{n}$ oticias/steam-enelaula/41064.html 